\title{
Acknowledgement to the Reviewers
}

The editors gratefully acknowledge the valuable contributions of the following reviewers to the evaluation of the papers submitted to Blood Purification.

M. Abramowitz
J. Agar
R. Amerling
F. Aregger
A.A. Arikan
G. Bayliss
A. Bellasi
G. Boriani
M. Bossola
E.A. Brown
J. Burton
S. Büttner
C. Campana
V. Campese
I. Campos
B. Canaud
A. Cases
D. Celdran-Bonafonte
C.T. Chan
K. Chatterjee
C. Chazot
Y. Cho
M.D. Churchwell
G. Cianciolo
G. Cohen
C. Confavreux
B. Cullis
G. D’Arrigo
A. Davenport
A. Deep
L. del Vecchio
L. Di Lullo
M. Dimopoulos
B. Ene-Iordache
R. Fagugli
I. Favia
A. Figueiredo
D.

M. Abramowitz

G. Forneris

L.G. Forni

F. Galli

H. Geng

M. Ghannoum

C. Gil

S.L. Goldstein

D. Gong

T.-J. Guan

M. Haase

N. Harbord

O. Heimburger

S. Hiremath

P. Honore

E.A. Hoste

J. Hudson

F. Husain-Syed

J. Ibeas

M. Javaid

A. Kakajiwala

A. Kazory

F. Keller

P. Kerr

J.T. Kielstein

D. Kirmizis

A. Koratala

D. Krieter

G. La Manna

L. Labriola

G. Landoni

M. Leite Jr.

E. Lerma

Y. Li

P. Liaveri

B. Lindholm

E. Lindley

R.M. Lindsay
F. Locatelli

C. Lomonte

J.A. Lopes

K.-C. Lu

$\mathrm{X} . \mathrm{Lu}$

N. Luo

F. Maduell

R. Malhotra

J. Malik

P. Malindretos

J. Malyszko

E. Mancini

C. Manno

D. Marcelli

P. Mark

A. Marn Pernat

I. Masakane

M. Sindhoora Mavuram

M. Meola

M. Messa

P. Messa

M. Migliori

T. Moraes

L. Morrone

A. Nayak

M. Ostermann

V. Panichi

E. Paoletti

G. Pertosa

E. Porrini

J. Potier

J. Raimann

A. Remuzzi

L. Renhua

Z. Ricci

J. Roberts

L. Rosales
A. Rosati

M. Rosner

Y. Sakamoto

F. Saliba

P. Saudan

R. Schindler

R. Schmitt

D. Schneditz

Y. Shi

A. Sikole

D. Soares

N. Srisawat

B.G. Stegmayr

P. Susantitaphong

Y. Taniyama

J.E. Tattersall

I. Teitelbaum

J. Teng

C. Tetta

D. Tomescu

J.H.M. Tordoir

S. Uchino

A. Valika

A. van Ballegooijen

F. van der Sande

B. van Jaarsveld

J. Van Sickle

G. Villa

V. Wadhwa

J. Winchester

J. Xie

F. Yang

W. Yu

M. Zhang

L. Zuo 